\title{
"L'éducation au développement durable de l'école au campus : enjeux pédagogiques et pratiques sociales dans les établissements d'enseignement »
}

\section{Compte rendu de colloque (Albi, 25-27 juin 2008)}

\author{
Odile Blanchard \\ Économiste, Université Pierre-Mendès-France, Laboratoire d'économie de la production et de l'intégration internationale (LEPII), \\ BP 47, 38040 Grenoble cedex 9, France
}

Ce colloque international a été coorganisé par le Centre universitaire de formation et de recherche (CUFR) JeanFrançois Champollion d'Albi et l'École des mines d'AlbiCarmaux, en partenariat avec l'Université Pierre-MendèsFrance de Grenoble, le Comité 21, la chambre régionale de commerce et d'industrie de Midi-Pyrénées, HEC Montréal, l'Association québécoise pour la promotion de l'éducation relative à l'environnement (AQPERE), la Coalition jeunesse Sierra du Canada. Au niveau local, de très nombreux acteurs ont contribué à la préparation du colloque : les associations étudiantes albigeoises, les parties prenantes de l'opération de maîtrise de l'énergie Écocampus, les membres du Centre permanent d'initiatives pour l'environnement du Tarn, les chargés de mission Agenda 21 et les représentants de différents écoétablissements d'enseignement de la région Midi-Pyrénées.

Le colloque d'Albi a fait suite à celui qui avait eu lieu un an auparavant à Trois-Rivières (Canada) : "Campus durables - Éco-Campus : mise en agenda et management environnemental dans les universités ». Ce premier colloque avait permis d'échanger sur les conditions de mise en agenda des démarches de développement durable dans les universités, sur la complexité du management de ces opérations qui impliquent de nombreux acteurs transcatégoriels (étudiants, enseignants, personnel nonenseignant) et sur la nécessité de pérenniser les initiatives. Le colloque d'Albi s'en est toutefois différencié par l'ampleur de l'évènement, les cibles visées, les objectifs et son organisation globale.

Auteur correspondant : Odile.Blanchard@upmf-grenoble.fr
Une des richesses de ce colloque a résidé dans le nombre et la diversité des participants. Ce sont environ 350 personnes de plusieurs pays francophones et d'horizons très diversifiés qui ont apporté leurs témoignages, leurs questionnements, leurs analyses. Les intervenants et les auditeurs incluaient non seulement les acteurs des universités, mais aussi ceux de l'enseignement primaire et secondaire, d'organisations non gouvernementales, de réseaux locaux, d'agences gouvernementales, etc. Se sont ainsi succédé à la tribune et rencontrés en sessions parallèles : enseignants-chercheurs, chercheurs, gestionnaires des universités et des grandes écoles, enseignants du premier et du second degré, proviseurs de lycées, coordinateurs pédagogiques développement durable au sein des inspections académiques, formateurs des Instituts universitaires de formation des maîtres (IUFM), animateurs d'associations ou de réseaux d'éducation au développement durable ou à l'environnement, chargés de mission développement durable au sein des départements ou des régions...

Les objectifs du colloque étaient : 1) de partager les expériences, les projets et les pratiques d'éducation au développement durable de l'école primaire au campus ; 2) de mettre en lumière les contraintes et les opportunités de coopération entre établissements et acteurs d'un territoire ; 3) de réfléchir sur les formations au développement durable, du point de vue de leur contenu, de la place à leur accorder et des besoins.

Le programme du colloque était structuré autour de ces trois axes, avec des conférences plénières introductives, puis des communications suivies de débats au sein 
d'ateliers se déroulant simultanément. Il a été enrichi le deuxième jour par un "colloque dans le colloque », mis en place par et à l'École des mines d'Albi-Carmaux, sur le thème "Vers de nouveaux métiers et un nouveau référentiel ». Par ailleurs, tout au long des trois journées, un écovillage sous chapiteau a permis aux participants de découvrir diverses initiatives d'associations visant à la diffusion de la culture du développement durable. Les thèmes principaux des échanges entre les participants ont été croqués par des artistes de la scène (clowns, slameur) et de la bande dessinée. Restitués en fin de séances de travail, ces brefs éclairages divertissants apportaient un regard sans concession sur les travaux des participants.

La logistique et l'intendance du colloque ont été assurées dans le souci de la moindre empreinte écologique, de la durabilité et de circuits courts pour la restauration : visioconférences pour certains intervenants, papier recyclé pour les cahiers des résumés des interventions, promotion des transports en commun et du covoiturage, tri sélectif des déchets, toilettes sèches, produits locaux issus de l'agriculture biologique... Les participants ont également pu prendre conscience de leur empreinte carbone, grâce à un outil de calcul des émissions de $\mathrm{CO}_{2}$ spécifique aux déplacements dans des colloques.

Concernant les présentations, elles ont été regroupées en trois ensembles, correspondant aux trois grands objectifs du colloque, à savoir : effectuer l'analyse de retours d'expérience ; montrer l'importance de la construction de réseaux, de partenariats ; explorer ce que peut recouvrir la formation au développement durable.

\section{Les retours d'expérience}

Les retours d'expérience ont été nombreux et variés. Ils ont permis à la fois de partager les initiatives engagées de l'école primaire au campus, de mettre en évidence les difficultés rencontrées ainsi que les facteurs de succès (ou d'échec), et de formuler diverses recommandations. Les échanges entre participants ont montré que les questions soulevées sont similaires, que ce soit à la maternelle, à l'école primaire, dans les collèges, les lycées ou les universités.

Les initiatives décrites correspondent le plus souvent à des projets de terrain tels que la mise en place de démarches et de plans d'actions du type Agenda 21, Écocampus, Campus vert, le déploiement d'un système de management environnemental, le développement d'outils de sensibilisation ou de diagnostic. Parmi les nombreuses présentations, on peut citer celle d'Isabelle Ottria, chargée de mission Agenda 21 scolaire à la direction régionale de l'agriculture et de la forêt de Midi-Pyrénées. I. Ottria a montré la dynamique qui a permis que l'expérimentation d'une démarche d'Agenda 21 menée dans un établissement agricole puisse être déployée ensuite dans quatorze autres établissements. David Wilgenbus, ingénieur d'études à l'École nationale supérieure (Paris), a présenté le projet d'éducation à l'environnement pour un développement durable «Le climat, ma planète... et moi ! » destiné aux classes de cycle 3 de l'école primaire. Odile Blanchard, maître de conférences en économie à 1'Université Pierre-Mendès-France (Grenoble), a exposé la méthodologie pour construire un inventaire des émissions de gaz à effet de serre dans un établissement d'enseignement et montré en quoi c'est à la fois un outil pour le diagnostic, l'action, le pilotage, la sensibilisation et la pédagogie.

Les initiatives peuvent être impulsées au départ par des élèves ou des étudiants dans une démarche bottomup ou, à l'inverse, par le haut (chef d'établissement, par exemple) dans une approche top-down. Elles peuvent être animées par un ou plusieurs coordinateurs. Les raisons de leur émergence sont multiples et dépendent en particulier des acteurs initiaux : le militantisme motivera généralement les initiatives étudiantes, tandis que ce sont la volonté d'exemplarité, la volonté de développer des pratiques conformes aux discours, aux valeurs mises en avant dans l'éducation au développement durable et la volonté de créer des effets d'entraînement qui primeront dans une approche top-down. Dans tous les cas, ces initiatives mènent à un décloisonnement des acteurs : le personnel administratif, les enseignants, les élèves (ou étudiants) sont appelés à travailler ensemble en interne et à rencontrer de nombreux partenaires externes (voir plus bas sur ce dernier point).

Les difficultés rencontrées concernent à la fois le manque de motivation et d'implication de certains acteurs, la résistance au changement de comportement, les barrières disciplinaires, la complexité de l'objet développement durable malgré son galvaudage, mais aussi le caractère temporaire inéluctable de l'implication des élèves ou des étudiants (de par leur séjour éphémère dans les établissements). Face à ces difficultés, l'adhésion clairement affichée du chef d'établissement (primaire, secondaire, supérieur), ainsi que l'énergie et la motivation des coordinateurs constituent les ingrédients nécessaires mais non suffisants - au succès des démarches.

Les recommandations issues des retours d'expérience portent essentiellement sur la nécessité de mettre en place les conditions de pérennisation des actions engagées (nomination d'un chargé de mission, passage de relais effectif entre élèves ou étudiants, officialisation/certification de la démarche, etc.), la nécessité d'appropriation de la démarche par les acteurs concernés pour une adhésion au projet (adaptation du projet au contexte) et la nécessité de faire participer les intéressés (création d'une instance d'animation dédiée au projet...). En s'appuyant sur l'expérimentation déployée sur le campus universitaire d'Albi pour la maîtrise de l'énergie, Marie-Christine Zelem, maître de conférences en sociologie au CUFR 
Jean-François Champollion d'Albi, et Stéphane Arditi, sociologue de l'équipe de recherche technologique «Services publics et économies d'énergie durables » de ce même CUFR, ont souligné l'intérêt d'adopter une approche sociotechnique associant changements techniques et modes de gouvernance pertinents pour mettre en place les changements de façon pérenne.

\section{Les réseaux et les partenariats}

Concernant le deuxième point focal du colloque, les communications ont mis en évidence les synergies tirées de l'ancrage territorial des démarches développement durable des établissements d'enseignement. Les collectivités territoriales, les associations locales, les antennes locales des agences gouvernementales (comme celles de l'Ademe) leur apportent un appui précieux par leur expérience, leurs animateurs, leurs outils de sensibilisation ou de diagnostic, leur coordination des initiatives et éventuellement leur soutien financier. Réciproquement, les établissements peuvent mettre leurs compétences en œuvre auprès de partenaires locaux, par exemple dans les démarches de recherche-action.

Par ailleurs, les structures régionales de coordination de l'enseignement (telles que les directions régionales de l'agriculture pour l'enseignement agricole, les rectorats d'académie) permettent de capitaliser les expériences menées, de mutualiser les outils développés et de fédérer petit à petit de nouveaux établissements dans la démarche développement durable qu'ils ont impulsée. Emmanuel Bon, animateur régional « agriculture durable et développement durable» de la direction régionale de l'agriculture et de la forêt de Haute-Normandie, a expliqué comment onze partenaires institutionnels de Haute-Normandie ont monté, animé et coordonné un projet pédagogique d'éducation au développement durable de type Agenda 21, dans lequel sont désormais engagés plus de 90 établissements d'enseignement ou de formation de la région.

\section{Les formations}

Sur le troisième point focal du colloque, les participants ont tous convenu que l'éducation au développement durable est pluridisciplinaire et transversale par essence, qu'elle vise à la fois à acquérir des connaissances, à développer des compétences pratiques, à éduquer à la concertation et aux démarches participatives et à véhiculer des valeurs éthiques et une culture qui appellent des modifications de comportements. Pour toutes ces raisons, elle constitue un changement culturel majeur par rapport aux pratiques pédagogiques habituelles. Pourtant, les enseignants français y sont peu formés. En conséquence, ils sortent difficilement d'un fonctionnement disciplinaire, comme l'a conclu Caroline Leininger-Frezal, professeur relais pour l'académie de Lyon au Graine ${ }^{1}$ Rhône-Alpes, dans l'enquête qu'elle a réalisée sur les pratiques des enseignants en matière d'éducation à l'environnement vers un développement durable.

Lors du colloque dans le colloque «Vers de nouveaux métiers et un nouveau référentiel», il a été souligné que l'éducation au développement durable n'est pas une discipline à enseigner mais une démarche. Certains intervenants ont appelé à la création d'un référentiel en la matière, qui traiterait des savoirs, des compétences, des aptitudes et des comportements. Différents nouveaux métiers liés au développement durable ont été mis en avant, mais les participants ont convenu que les offres d'emploi dans ce domaine sont encore peu nombreuses.

En conclusion, le colloque a permis de rassembler et de faire dialoguer de multiples acteurs qui ne se rencontrent pas habituellement, alors qu'ils œuvrent tous dans le domaine de l'éducation au développement durable. Les débats ont été d'autant plus riches que les sessions parallèles ont été organisées de façon à décloisonner les « communautés ». La mutualisation des savoirs, des expériences et des leçons qui en ont été tirées a mis en évidence la complexité des démarches, la nécessité de dépasser les clivages habituels, qu'ils soient disciplinaires ou communautaires (élèves ou étudiants, enseignants, non-enseignants), pour s'orienter vers la transversalité disciplinaire et l'intégration des communautés. Certes, la dimension environnementale a été la plus abordée dans les présentations et les échanges entre participants. Mais les autres dimensions du développement durable ont aussi fait l'objet d'analyses et de débats².

À terme, une réflexion approfondie méritera d'être menée sur la distinction nécessaire entre éducation relative à l'environnement et éducation au développement durable. En conclusion du colloque, Lucie Sauvé, professeure au département d'éducation et pédagogie de l'Université du Québec à Montréal et titulaire dans cette institution de la chaire de recherche du Canada en éducation relative à l'environnement, a ouvert cette réflexion. Elle a souligné la richesse des théories et des pratiques construites au cours des dernière décennies dans le champ de l'éducation relative à l'environnement, face à un projet mondial d'éducation au développement durable dont la vision pragmatique et anthropocentriste entretient la rupture entre l'homme et la nature.

\footnotetext{
${ }^{1}$ Groupement régional pour l'animation et l'initiation à la nature et l'environnement. Chaque Graine regroupe par région les personnes (enseignants, animateurs...), les associations et les institutions qui s'impliquent dans l'éducation à l'environnement.

${ }^{2}$ Les communications du colloque sont disponibles en ligne à l'URL http://colloque-edd-2008.mines-albi.fr. Certaines ont fait l'objet d'un ouvrage dont la parution est prévue début 2010 : Zélem, M.-C., Blanchard, O., Lecomte, D. (Eds), Le Développement durable, de l'école au campus, Paris, L'Harmattan.
} 